\title{
Tailoring the magnetic anisotropy and domain patterns of sputtered TbFeGa alloys
}

\author{
R. Ranchal ，S. Fin , D. Bisero , C. Aroca
}

\begin{abstract}
A B S T R A C T
We report the magnetic anisotropy and domain configuration of cosputtered $\mathrm{TbFeGa}$ alloys. The layers were deposited from two targets with compositions $\mathrm{TbFe}_{2}$ and $\mathrm{Fe}_{3} \mathrm{Ga}$, respectively. The structural and magnetic properties do not only depend on the composition but also on the growth conditions. Alloys with the same composition but deposited using a DC or a pulsed power source in the $\mathrm{TbFe}_{2}$ target exhibit a different magnetic anisotropy. The perpendicular magnetic anisotropy, the size and topology of domain patterns can be tailored by changing the evaporation parameters of $\mathrm{TbFe}_{2}$. The width of the stripe domain increases from 235 to $835 \mathrm{~nm}$ when using the DC source in the $\mathrm{TbFe}_{2}$. We correlate this effect with $\mathrm{Tb}$ enrichment of the $\mathrm{Tb}_{x} \mathrm{Fe}_{1}$ x phases present in the samples.
\end{abstract}

\section{Introduction}

The control of the magnetic domain patterns together with their time and temperature stability are key issues for the development of new devices. Materials systems with perpendicular magnetic anisotropy (PMA) are of great interest because of their applications on patterned media for magnetic storage or in spintronic devices [1-3]. Systems exhibiting PMA have larger magnetic anisotropy energy and the domain configuration is more stable and uniform as compared to most of in-plane anisotropy materials [4]. For example, in spintronic devices with ultrahigh density, it is necessary to employ systems with an anisotropy energy constant equal or higher than $10^{7} \mathrm{erg} / \mathrm{cm}^{3}$ [3]. Nowadays, different approaches are analyzed to reach this high magnetic anisotropy, leading to various structures such as FePt and CoPt layers [5,6], multilayers comprising magnetic transition metals ( $\mathrm{Co}, \mathrm{Fe}, \mathrm{CoFe})$ and noble metals (Pt, $\mathrm{Pd}, \mathrm{Au}$ ) [7-9] or FeCoB layers with PMA [10].

Some years ago, $\mathrm{Tb}-\mathrm{Fe}$ alloys were greatly investigated because of their pretty large out-of-plane anisotropy constant that can reach $10^{7} \mathrm{erg} / \mathrm{cm}^{3}$ [11]. Some works on bulk TbFeGa alloys have focused on the magnetostrictive properties of these alloys [12,13] but recently, it has also been reported PMA in ternary TbFeGa alloys obtained by cosputtering from two targets with composition $\mathrm{TbFe}_{2}$ and $\mathrm{Fe}_{3} \mathrm{Ga}$, respectively [14]. In that work, a fixed DC power of $100 \mathrm{~W}$ was applied in the $\mathrm{TbFe}_{2}$ whereas in the $\mathrm{Fe}_{3} \mathrm{Ga}$ target the pulsed power source ranged between 60 and $120 \mathrm{~W}$. For these growth conditions, the PMA was observed in some of the studied compositions, $\mathrm{Tb}_{12} \mathrm{Fe}_{78} \mathrm{Ga}_{10}, \mathrm{~Tb}_{10} \mathrm{Fe}_{78} \mathrm{Ga}_{12}$, and $\mathrm{Tb}_{10} \mathrm{Fe}_{76} \mathrm{Ga}_{14}$, being found an anisotropy energy constant of at least $1 \times 10^{6} \mathrm{erg} / \mathrm{cm}^{3}$. In spite of the rather similar composition of all those previous alloys, the magnetic properties are quite different and the experimental results indicate that the presence of PMA depends on the composition of the $\mathrm{Tb}_{x} \mathrm{Fe}_{1-x}$ crystalline phases present in those samples. In the present work, we investigate the influence of the type of power source used in each sputtering target. We show that in TbFeGa alloys the magnetic domain patterns can be tailored by means of not only the composition but also the power source.

\section{Experimental techniques}

Samples were deposited at room temperature on $5 \times 4 \mathrm{~mm}^{2}$ glass substrates. Two targets with a nominal composition of $\mathrm{TbFe}_{2}$ and $\mathrm{Fe}_{3} \mathrm{Ga}$ were employed to deposit by cosputtering the alloys. The cosputtering process was performed in the oblique incidence, being the angle of incidence between each target and the substrate of about 25 degrees $[14,15]$ and the distance between the targets and the substrate of $15 \mathrm{~cm}$. Two series of samples have been obtained depending on the type of power source (DC or pulsed) it is used in each target. In series A, a fixed $\mathrm{DC}$ power of $100 \mathrm{~W}$ is applied in the $\mathrm{Fe}_{3} \mathrm{Ga}$ target and different compositions are achieved by modifying the pulsed power from 60 to $120 \mathrm{~W}$ in the $\mathrm{TbFe}_{2}$ target. In series $\mathrm{B}$, the DC source is applied at a fixed power of $100 \mathrm{~W}$ in the $\mathrm{TbFe}_{2}$ target, whereas the pulsed power ranged between 80 and $120 \mathrm{~W}$ in the $\mathrm{Fe}_{3} \mathrm{Ga}$. In all cases, the frequency $(25 \mathrm{kHz})$ and the dead-time $(5 \mu \mathrm{s})$ were fixed parameters in the pulsed source. The thickness of the TbFeGa layers was around $250 \mathrm{~nm}$. Mo layers 
$(20 \mathrm{~nm})$ were used as buffer and capping layers for all the samples. They were grown with a DC power of $90 \mathrm{~W}$. The Ar pressure was $2 \times 10^{-3}$ mbar to evaporate all the layers: buffer, capping, and ternary TbFeGa alloys.

The composition of the samples was analyzed by means of the Energy Dispersive X-ray Spectroscopy (EDS) in a Leica 440 SEM microscope operated at $10 \mathrm{kV}$ and $2 \mathrm{nA}$. $\theta-2 \theta \mathrm{X}$-ray diffractometry patterns were measured in the BraggBrentano configuration. At room temperature, in-plane and out-of-plane hysteresis loops were carried out in a vibrating sample magnetometer (VSM). In the VSM we can rotate the sample being possible to measure the in-plane loops at different angles between the applied magnetic field and the in-plane reference direction, the long side of the substrates. The same routine as in previous works was used for the field-cooled (FC) curves performed in a superconducting quantum interference device (SQUID) magnetometer [16]. Prior to measuring the temperature dependence of the magnetization, the sample was first cooled from room temperature to $5 \mathrm{~K}$ under a saturation field of $2 \mathrm{k}$ Oe and then, the FC curves were recorded with an applied magnetic field of 100 Oe during the warming-up.

Magnetic Force Microscopy (MFM) images were recorded by a Digital Instruments Nanoscope Illa, using the phase detection mode, i.e., monitoring the cantilever's phase of oscillation while the magnetic tip was scanning the sample surface at a distance of $80 \mathrm{~nm}$ on the average (lift mode) [17,18]. Commercially available ferromagnetic CoCr tips were used. The MFM measurements were performed both at remanence and in-field (applying an external magnetic field lying in the plane of the film, with an intensity of $0.8 \mathrm{k} \mathrm{Oe}$ ). In order to exclude the influence of the tip on the magnetic state of the sample, we used different scanning directions and tip to sample distances, obtaining the same results with different operating conditions.

\section{Results and discussion}

We have measured the composition of all the samples by the EDS technique (Table 1). The TbFeGa alloys were compositionally homogeneous and we have not observed any of the compositional fluctuations reported in the work by Jiang et al. [13]. Nevertheless, it is important to remark that different growth conditions and different compositions have been studied in each work. In Ref. [13] there are investigated samples of FeGa alloys doped with contents of $\mathrm{Tb}$ between 0.2 and 0.8 obtained by arc-melting whereas in our work, we deposit thin films with a composition around $\mathrm{Tb}_{10} \mathrm{Fe}_{76} \mathrm{Ga}_{14}$ by the cosputtering technique. With this technique it is possible to tune the composition of the samples by means of the growth power. The decrease (increase) of pulsed power in the $\mathrm{TbFe}_{2}\left(\mathrm{Fe}_{3} \mathrm{Ga}\right)$ target raises the $\mathrm{Ga}$ content in the TbFeGa alloys. Moreover, the use of a DC or a pulsed power source in each target allows to obtain the same composition from different growth conditions (Table 1).

As in the previous work on sputtered TbFeGa alloys [14], the Xray diffraction patterns of the two series of samples only exhibit one main diffraction peak at around $2 \theta \sim 40.5^{\circ}$ (Fig. $1 \mathrm{a}$ and $\mathrm{b}$ ). This peak is close to the most intense reflection of the $\mathrm{TbFe}_{2}$ Laves phase, the [113] that appears at $2 \theta=40.74^{\circ}$. We have not found evidences of any other peak close to any of those previously reported in $\mathrm{Fe}_{1-y} \mathrm{Ga}_{y}$ or TbFeGa alloys and then, the experimentally observed diffraction peak can be related to the presence of crystalline $\mathrm{Tb}_{x} \mathrm{Fe}_{1-x}$ phases with a structure close to the cubic $\mathrm{TbFe}_{2}$. In any case, the intensity of this peak is low and we cannot consider our samples as epitaxial.

In Fig. 2a we present the diffraction angle as a function of the Ga content for the two series of samples. In both series, the decrease of the Ga content shifts the diffraction peak towards the theoretical diffraction angle of the $\mathrm{TbFe}_{2}$ cubic structure $\left(40.74^{\circ}\right)$. Nevertheless, for samples with the same composition the position of the diffraction
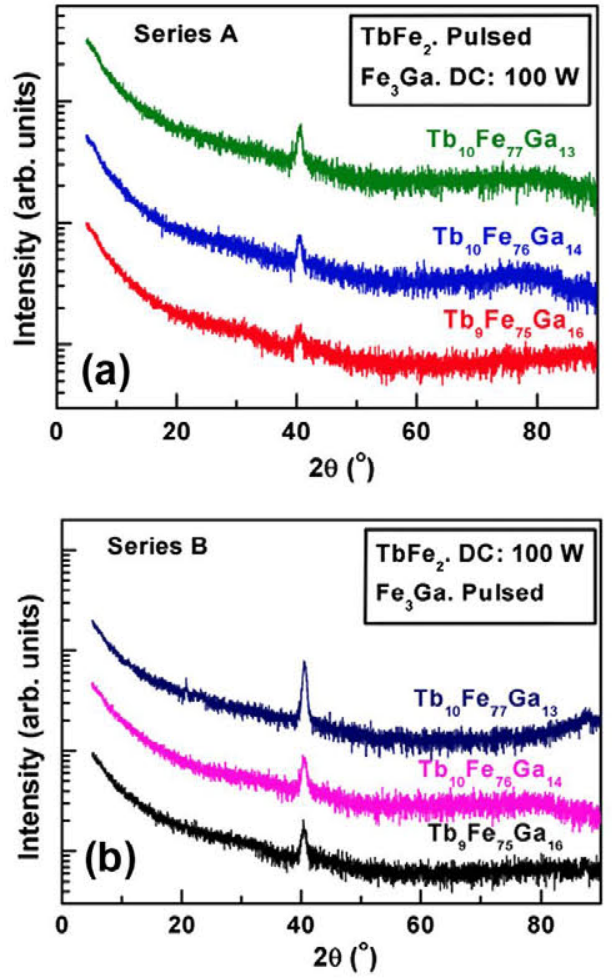

Fig. 1. X-ray diffraction patterns of $\mathrm{Tb}_{x} \mathrm{Fe}_{1-x} \mathrm{Ga}$ films with different compositions. In each curve is displayed the composition of the sample. (a) Series A in which the pulsed source is applied in the $\mathrm{TbFe}_{2}$ target and (b) series B in which the pulsed source is applied in the $\mathrm{Fe}_{3} \mathrm{Ga}$ target. Curves are vertically shifted for clarity.

peak depends on the growth conditions, i.e. the type of power source. The peak appears closer to $40.74^{\circ}$ in layers from set $B$ (Fig. 2a) revealing a structure more similar to the $\mathrm{TbFe}_{2}$ in that series of samples. We have calculated the lattice parameter from the diffraction scans considering a cubic structure (Fig. 2b). In all cases, the lattice parameter is higher than the theoretical value of $\mathrm{TbFe}_{2}$ (7.341 $\AA$ ) and in both series depends on the Ga content. Mo grows as a pollycrystal on glass and a possible influence of the lattice mismatch between Mo and TbFeGa is not obvious to analyze. In any case, the influence of the Ga content and the growth conditions on the lattice parameter (Fig. 2b) indicates that these two parameters have a stronger influence on it than a hypothetical lattice mismatch between the Mo buffer and the TbFeGa layers.

The type of power source also appears to be of relevance as samples with the same composition but deposited in different ways exhibit a different intensity for the diffraction peak and different lattice parameters (Figs. 1 and 2b). On one hand, the increase of the $\mathrm{Ga}$ content promotes the decrease of the peak intensity suggesting that Ga doping disturbs the crystalline structure (Fig. 1). The peak intensity is higher in set $\mathrm{B}$ in comparison to A revealing that the use of the $\mathrm{DC}$ power source to evaporate the $\mathrm{TbFe}_{2}$ promotes layers with a higher structural quality. On the other, layers of set $B$ have a lattice parameter closer to the

Table 1

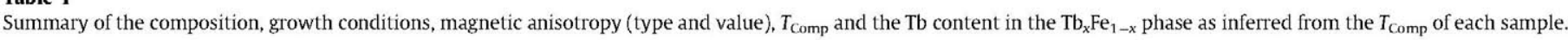

\begin{tabular}{|c|c|c|c|c|c|}
\hline Composition & Growth conditions & Magnetic anisotropy $\left(\mathrm{erg} / \mathrm{cm}^{3}\right)$ & $T_{\text {Comp }}(\mathrm{K})$ & Tb in the $\mathrm{Tb}_{x} \mathrm{Fe}_{1-x}$ (at.\%) & \\
\hline $\mathrm{Tb}_{10} \mathrm{Fe}_{77} \mathrm{Ga}_{13}$ & 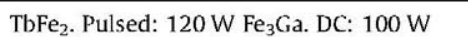 & $\mathrm{PMA} \geqslant 1.5 \times 10^{6}$ & $\geqslant 300$ & $\geqslant 28$ & Series A \\
\hline $\mathrm{Tb}_{10} \mathrm{Fe}_{76} \mathrm{Ga}_{14}$ & $\mathrm{TbFe}_{2}$. Pulsed: $100 \mathrm{~W} \mathrm{Fe}{ }_{3} \mathrm{Ga}$. DC: $100 \mathrm{~W}$ & Weak PMA $0.6 \times 10^{6}$ & 176 & 22 & \\
\hline $\mathrm{Tb}_{9} \mathrm{Fe}_{75} \mathrm{Ga}_{16}$ & $\mathrm{TbFe}_{2}$. Pulsed: $60 \mathrm{~W} \mathrm{Fe} \mathrm{Fa}_{3}$. Pulsed: $100 \mathrm{~W}$ & In-plane $0.5 \times 10^{6}$ & 31 & 18 & \\
\hline $\mathrm{Tb}_{10} \mathrm{Fe}_{77} \mathrm{Ga}_{13}$ & $\mathrm{TbFe}_{2}$. DC: $100 \mathrm{~W} \mathrm{Fe} \mathrm{Ga}_{3}$ Pulsed: $80 \mathrm{~W}$ & $\mathrm{PMA} \geqslant 1.5 \times 10^{6}$ & $\geqslant 300$ & $\geqslant 28$ & Series B \\
\hline $\mathrm{Tb}_{10} \mathrm{Fe}_{76} \mathrm{Ga}_{14}$ & $\mathrm{TbFe}_{2}$. DC: $100 \mathrm{~W} \mathrm{Fe} \mathrm{Fa}_{3}$ Pulsed: $100 \mathrm{~W}$ & PMA $1.0 \times 10^{6}$ & $\geqslant 300$ & $\geqslant 28$ & \\
\hline $\mathrm{Tb}_{9} \mathrm{Fe}_{75} \mathrm{Ga}_{16}$ & $\mathrm{TbFe}_{2}$. DC: $100 \mathrm{~W} \mathrm{Fe}$ Ga. Pulsed: $120 \mathrm{~W}$ & In-plane $0.4 \times 10^{6}$ & 15 & 16 & \\
\hline
\end{tabular}



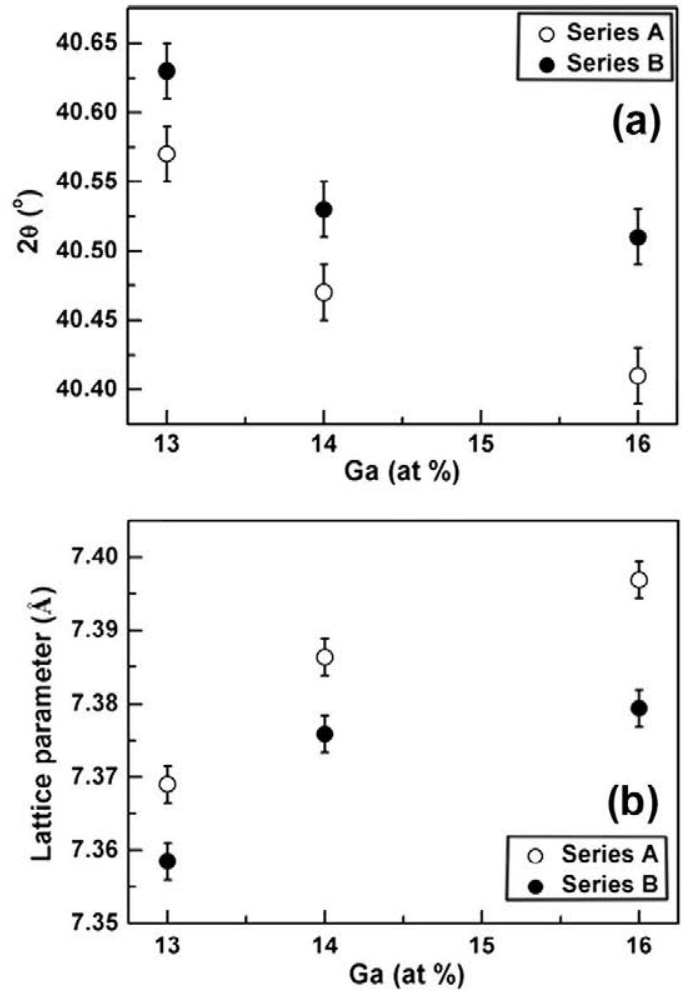

Fig. 2. (a) Position of the diffraction peak as a function of the Ga content for the two series of samples: series A (O) and series B ( $)$. (b) Lattice parameter as a function of the Ga content for the two series of samples: series A (O) and series B (-).

theoretical value of $\mathrm{TbFe}_{2}(7.34 \AA)$ that indicates a $\mathrm{Tb}_{x} \mathrm{Fe}_{1-x}$ crystalline phase more similar to the Laves phase (Fig. 2b).

We have determined the type of magnetic anisotropy at room temperature following the methodology used in a previous work [14]. A sample will show PMA if two conditions are fulfilled: (a) it is magnetically isotropic in the sample plane, and (b) the outof-plane direction is an easy axis in comparison to any direction in the sample plane. In Table 1 we have summarized the magnetic anisotropy found in each sample following this procedure. In the two series of samples, the magnetic anisotropy evolves from in-plane to PMA as the Ga content is decreased. In the VSM, the maximum possible applied magnetic field was of $12.5 \mathrm{k}$ Oe and in some cases, the hysteresis loops could not be saturated. In Table 1 we present the inferred values for all the studied samples although the exact anisotropy constant could not always exactly be evaluated. In samples showing a clear PMA, we have inferred an anisotropy constant of at least $1 \times 10^{6} \mathrm{erg} / \mathrm{cm}^{3}$, a value of the same order of magnitude as compared to previously reported in TbFe $[11,19,20]$. Nevertheless, the growth conditions also have an effect on the anisotropy. The $\mathrm{Tb}_{10} \mathrm{Fe}_{76} \mathrm{Ga}_{14}$ alloy does show a different anisotropy in each set of samples. In Fig. 3 we present the VSM hysteresis loops recorded with the applied magnetic field perpendicular and in the sample plane for this alloy. The magnetization curves indicate that the alloy from series A exhibits a weak PMA (Fig. 3a) whereas the sample from series $B$ shows a distinct PMA Fig. 3b). Also, the inferred anisotropy energy constant is different in each sample (Table 1). Therefore, the magnetic anisotropy can be tuned by means of both the composition and the type of power source (growth conditions).

In spite of being observed signatures of PMA in the hysteresis loops, the magnetization curves also show a second magnetic phase with a low coercivity (inset of Fig. 3a and b). Therefore, in order to gain a deeper insight into the PMA properties of TbFeGa alloys we have performed Magnetic Force Microscopy (MFM)
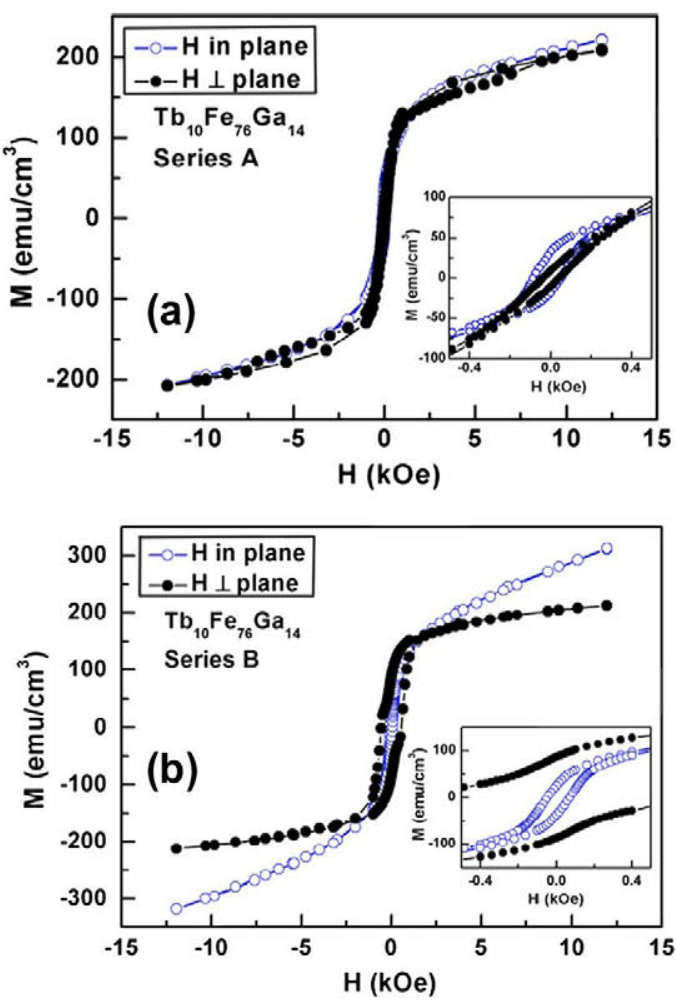

Fig. 3. Room temperature hysteresis loops of the $\mathrm{Tb}_{10} \mathrm{Fe}_{76} \mathrm{Ga}_{14}$ measured with the applied magnetic field perpendicular (๑) and in the sample plane (O). (a) Alloy from series A and (b) alloy from series B. In the insets of each figure it is shown the low field region.

measurements on all the samples described in this paper. A surface area without gross topographic features (thus representative for most part of the film) has been carefully selected for each measurement. Films with high PMA usually show up/down stripe domains, which cause black and white contrast in MFM images, giving information on the orientation of the sample stray field gradient (and hence of the underlying magnetic moments) with respect to the magnetic dipole of the tip. The domain size is determined by an equilibrium between domain wall energy and magnetostatic energy.

The MFM results confirm the overall magnetic anisotropy data summarized in Table 1. In particular, Fig. 4 reports the out of plane magnetic signal recorded at remanence from the two samples described in Fig. 3. The MFM image corresponding to the sample from series A (Fig. 4a) displays stripe domains organized in labyrinthine patterns. A similar structure is shown by the sample from series B, but in this case the stripe domains appear wider and the measured perpendicular signal is more intense.

We have applied a bidimensional Fast Fourier Transform (FFT) to both images in order to determine a prevalent wavelength of the magnetic structure. As evidenced in Fig. 5 the maximum of the 2D power spectral density (PSD) is $235 \mathrm{~nm}$ for series A (Fig. 5a) and $835 \mathrm{~nm}$ for series B (Fig. 5b). Moreover, the stripe domains pattern disappears when a 800 Oe in-plane magnetic field is applied to sample from series A, confirming the VSM results, which are consistent with the presence of a weak PMA (Fig. 3a). On the other hand, the same experiment performed on sample from series B points out a strong residual MFM signal, comparable with the one observed at remanence. Therefore, we can say that a magnetic field of 800 Oe intensity can reorient the magnetic moments from out of plane to in plane, only in the sample of series A. This does not occur for the one of series B, due to the strong out of plane magnetic anisotropy of this film. Then, in spite of the same 

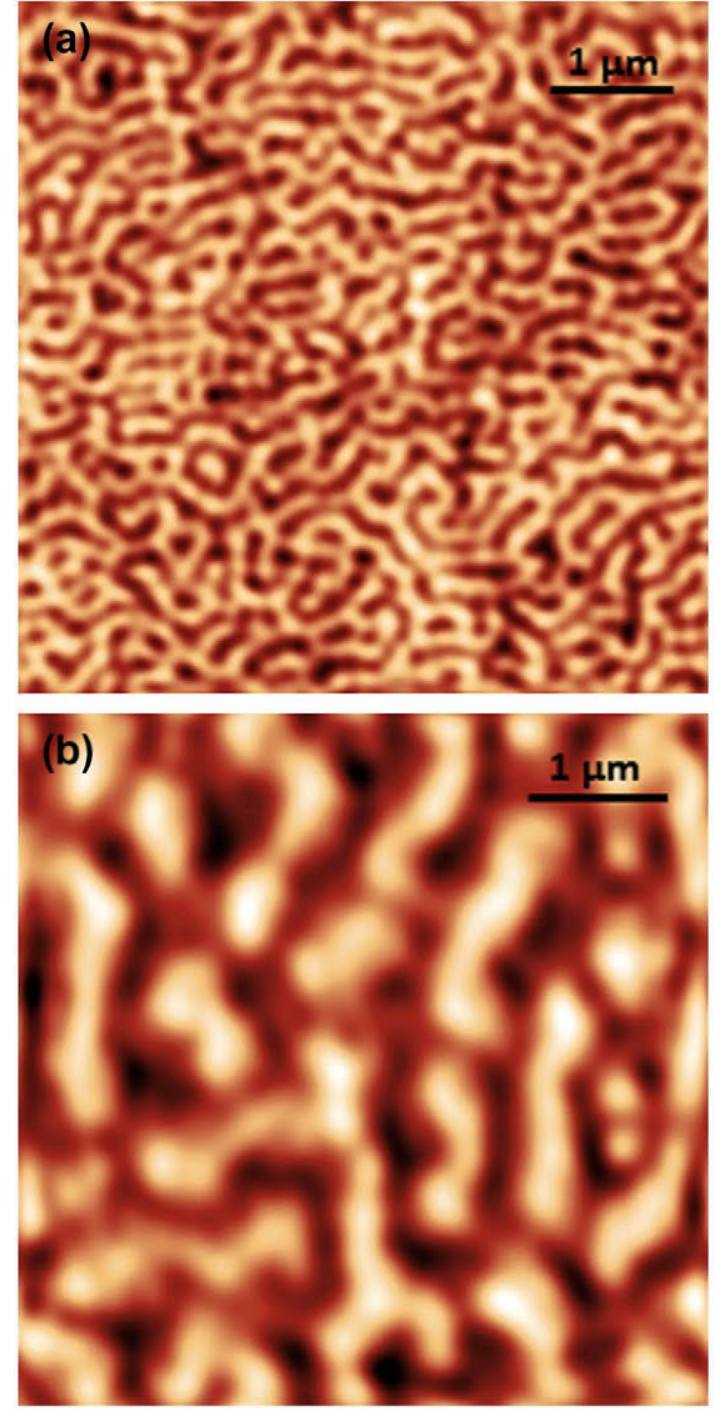

Fig. 4. MFM signal recorded at remanence from the sample $\mathrm{Tb}_{10} \mathrm{Fe}_{76} \mathrm{Ga}_{14}$. (a) Alloy from series $A$ and (b) alloy from series $B$.

composition $\left(\mathrm{Tb}_{10} \mathrm{Fe}_{76} \mathrm{Ga}_{14}\right.$ ) the PMA is enhanced in the alloy deposited under the growth conditions of series $\mathrm{B}$.

In the samples in which the VSM hysteresis loops indicated PMA, we have clearly observed stripe magnetic domains in the MFM images. Then, once the MFM has confirmed the existence of PMA, we can come back to the magnetometry results in order to find the origin of the low coercivity behavior (Fig. 3, insets). Low temperature hysteresis loops of cosputtered TbFeGa alloys deposited under the growth conditions of series B have been previously reported [14]. In agreement with the room temperature VSM measurements, the SQUID loops confirm the existence of two magnetic phases with distinct coercive fields. The hard magnetic phase is correlated to the $\mathrm{Tb}_{x} \mathrm{Fe}_{1-x}$ phase with a structure close to the $\mathrm{TbFe}_{2}$ as indicated by the XRD results, the inferred values of the anisotropy energy constant and the SQUID data presented in [14].

FC curves can provide further information about the structural properties. In materials systems comprised of heavy rare earths and magnetic transition metals it is observed a minimum (zero magnetization) in the FC curve generally denoted as the Compensation temperature ( $T_{\mathrm{Comp}}$ ) due to the antiferromagnetic coupling between these two type of atoms [21]. In our ternary alloys, the existence of $T_{\text {Comp }}$ in the FC curves can be due to the $\mathrm{Tb}_{x} \mathrm{Fe}_{1-x}$ crystalline phases detected by XRD. Furthermore, as the correlation between the composition of $\mathrm{Tb}_{x} \mathrm{Fe}_{1-x}$ alloys and the $T_{\text {Comp }}$ is
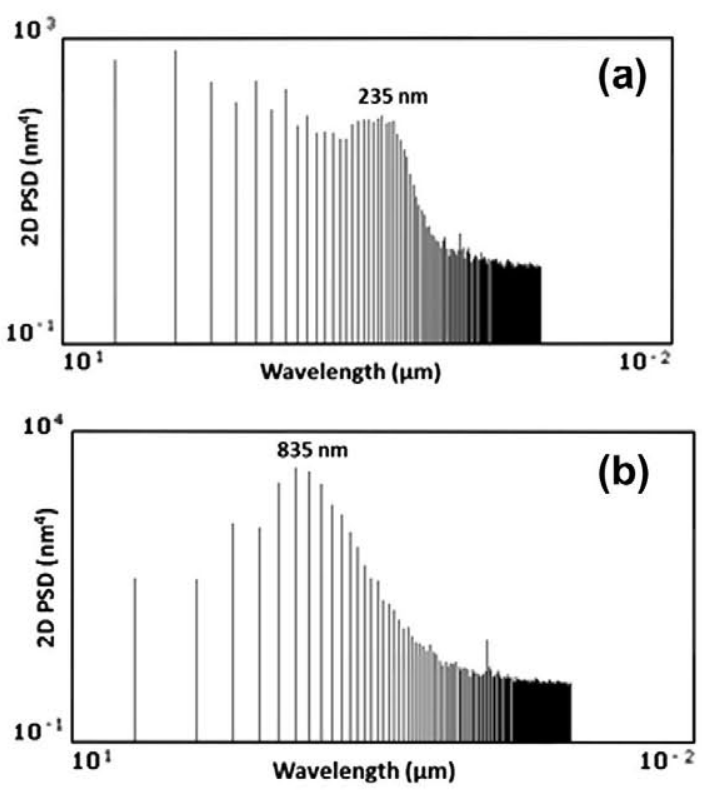

Fig. 5. 2D power spectral density obtained by Fast Fourier Transform (FFT) of the MFM images. (a) Alloy from series A and (b) alloy from series B.

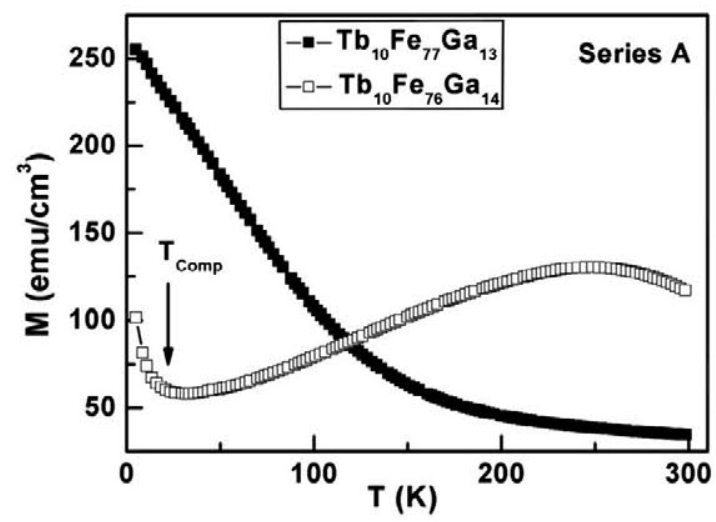

Fig. 6. FC curves of two alloys from series $\mathrm{A}$, ( $\square) \mathrm{Tb}_{10} \mathrm{Fe}_{77} \mathrm{Ga}_{13}$ and ( $\square$ ) $\mathrm{Tb}_{10} \mathrm{Fe}_{76} \mathrm{Ga}_{14}$.

already known [22], it is possible to analyze the Tb content in those former phases by using the $T_{\text {Comp }}$ [14]. Nevertheless, it is noteworthy that the presence of two ferromagnetic orderings in our samples avoids a zero magnetization in the $T_{\text {Comp. }}$. Although the $\mathrm{Tb}_{x} \mathrm{Fe}_{1-x}$ is the origin of the $T_{\text {Comp }}$, there is another second magnetic phase that has a contribution for the magnetization at the $T_{\text {Comp. }}$. Then, in some TbFeGa alloys we have observed a clear minimum in the FC curve, but not a zero magnetization, from which it is possible to determine the $T_{\text {Comp }}$ as it can be observed in Fig. 6 . In other alloys, it is not observed any minimum in the studied temperature range being the $T_{\text {Comp }}$ above room temperature (Fig. 6).

In Table 1 , we present the $T_{\text {Comp }}$ measured in the FC curves and the $\mathrm{Tb}$ content (at.\%) in the $\mathrm{Tb}_{x} \mathrm{Fe}_{1-x}$ phases we have inferred from them. We found a strong PMA in those samples with a $T_{\text {Comp }}$ higher than $300 \mathrm{~K}$, i.e. samples comprised of $\mathrm{Tb}_{x} \mathrm{Fe}_{1-x}$ with a Tb content equal or higher than 28 at.\%. In the two series, the $T_{\text {Comp }}$ decreases with the Ga content but the data from Table 1 also shows that it also depends on the growth conditions. The $\mathrm{Tb}_{10} \mathrm{Fe}_{76} \mathrm{Ga}_{14}$ alloy from series $A$, that shows a weak PMA, has a $T_{\text {Comp }}$ of $176 \mathrm{~K}$ that indicates a $\mathrm{Tb}$ content of around 22 at.\% in the $\mathrm{Tb}_{x} \mathrm{Fe}_{1-x}$. Nevertheless, the alloy from series B has a $T_{\text {Comp }}$ higher than $300 \mathrm{~K}$ that represents a $\mathrm{Tb}$ content equal or higher than $28 \%$. This sample from set $B$ exhibits a strong PMA with a wider stripe domain configuration 
$(835 \mathrm{~nm})$ than sample from series A $(235 \mathrm{~nm})$. The different content of $\mathrm{Tb}$ in the $\mathrm{Tb}_{x} \mathrm{Fe}_{1-x}$ phases present in ternary alloys with the same composition reflects the influence of the type of power source on the structural properties. This different structure seems to be the origin of the different magnetic anisotropy in those samples.

In samples from series $B$ the higher $T_{\text {Comp }}$ values (Table 1 ) and closer values of the lattice parameter to the theoretical values of the $\mathrm{TbFe}_{2}$ (Fig. 2b) points to a crystalline $\mathrm{Tb}_{x} \mathrm{Fe}_{1-x}$ phase similar to the Laves phase. In those samples, the PMA is more relevant as indicated by the magnetometry and the MFM data. The $\mathrm{Tb}_{x} \mathrm{Fe}_{1-x}$ is the hard magnetic phase that is related to the PMA as we have already discussed. $\mathrm{Ga}$ and $\mathrm{Fe}$ atoms that are not in this phase must form another highly $\mathrm{Fe}_{1-y} \mathrm{Ga}_{y}$ disordered phase (amorphous-like) as it cannot be detected by means of XRD. Due to it is highly disorder state, it is difficult to determine its composition and it can even be non-stoichiometric. However, this phase can account for the low coercivity behavior observed in the magnetometry results (insets Fig. $3 \mathrm{~b}$ ) as this type of alloys show coercive fields of around tenths of Oe [23]. The formation of this second magnetic phase depends on the type of power source. When the DC source is used to evaporate the $\mathrm{TbFe}_{2}$, the crystalline $\mathrm{Tb}_{x} \mathrm{Fe}_{1-x}$ has a higher Tb content and the PMA is enhanced. On the contrary, when the DC source is applied in the $\mathrm{Fe}_{3} \mathrm{Ga}$ target, the low coercivity magnetic phase is promoted (insets of Fig. 3), and the PMA is reduced. Therefore, the improvement of the out of plane component because of the use of a DC source in the $\mathrm{TbFe}_{2}$ target is due to the $\mathrm{Tb}$ enrichment of the $\mathrm{Tb}_{x} \mathrm{Fe}_{1-x}$ phases with a structure close to the Laves phase present in the TbFeGa alloys. The type of power source used on each sputtering target can be used to tune the magnetic anisotropy in the TbFeGa alloys.

\section{Conclusions}

We have studied the magnetic anisotropy of cosputtered TbFeGa alloys with different compositions and deposited under different growth conditions. The existence of PMA in these TbFeGa alloys is not only related to the composition but also to the type of power source used during the cosputtering process in each target. The increase of $\mathrm{Tb}$ in the $\mathrm{Tb}_{x} \mathrm{Fe}_{1-x}$ phases enhances the PMA being also possible to tune the magnetic anisotropy by means of the growth conditions. In particular, the evaporation of $\mathrm{TbFe}_{2}$ by means of a DC source enhances the out of plane component of the magnetization.

\section{Acknowledgements}

This work has been financially supported through the project MAT2011-28751-C02 of the Spanish Ministry of Science and Innovation and the project PRIN 2010ECA8P3 "DyNanoMag" of the Italian Ministry of Education, University and Research. We are in debt with Dr. D. Maestre (UCM) for the EDS compositional measurements. We thank "CAI Difracción de rayos-X" of UCM for the Xray diffractometry measurements and "CAI Técnicas Físicas" also from UCM for SQUID characterization.

\section{References}

[1] A.J. Schellekens, A. van den Brink, J.H. Franken, H.J.M. Swagten, B. Koopmans, Nat. Commun. 3 (2012) 1848

[2] T. Zhu, Y. Yang, R.C. Yu, H. Ambaye, V. Lauter, J.Q. Xiao, Appl. Phys. Lett. 100 (2012) 202406.

[3] S. Mangin, D. Ravelosona, J.A. Katine, M.J. Carey, B.D. Terris, E.E. Fullerton, Nat. Mater. 5 (2006) 210

[4] R. Sbiaa, H. Meng, S.N. Piramanayagam, Phys. Status Solidi RRL 5 (2011) 413.

[5] F. Casoli, F. Albertini, L. Nasi, S. Fabbrici, R. Cabassi, F. Bolzoni, C. Bocchi, Appl. Phys. lett. 92 (2008) 142506.

[6] H. Sato, T. Shimatsu, Y. Okazaki, H. Muraoka, H. Aoi, S. Okamoto, O. Kitakami, J. Appl. Phys. 103 (2008) 07E114.

[7] B. Carvello, C. Ducruet, B. Rodmacq, S. Auffret, E. Gautier, G. Gaudin, B. Dieny, Appl. Phys. Lett. 92 (2008) 102508.

[8] Y. Ochiai, S. Hashimoto, K. Aso, IEEE Trans. Magn. 25 (1989) 3755.

[9] K. Yakushiji, T. Saruya, H. Kubota, A. Fukushima, T. Nagahama, S. Yuasa, K. Ando, Appl. Phys. Lett. 97 (2010) 232508.

[10] S. Ikeda, K. Miura, H. Yamamoto, K. Mizunuma, H.D. Gan, M. Endo, S. Kanai, J. Hayakawa, F. Matsukura, H. Ohno, Nat. Mater. 9 (2010) 721.

[11] F. Hellman, A.L. Shapiro, E.N. Abarra, R.A. Robinson, R.P. Hjelm, P.A. Seeger, J.J. Rhyne, J.I. Suzuki, Phys. Rev. B 59 (1999) 11408.

[12] Y.J. Tang, H.L. Luo, N.F. Gao, Y.Y. Liu, S.M. Pan, Appl. Phys. Lett. 66 (1995) 388.

[13] L. Jiang, J. Yang, H. Hao, G. Zhang, S. Wu, Y. Chen, O. Obi, T. Fitchorov, V.G. Harris, Appl. Phys. Lett. 102 (2013) 222409.

[14] R. Ranchal, V. Gutiérrez-Díez, Thin Solid Films 534 (2013) 557.

[15] M. Romera, R. Ranchal, D. Ciudad, M. Maicas, C. Aroca, J. Appl. Phys. 110 (2011) 083910.

[16] R. Ranchal, V. González-Martín, J. Appl. Phys. 110 (2011) 053901.

[17] P. Vavassori, V. Bonanni, A. Busato, D. Bisero, G. Gubbiotti, A.O. Adeyeye, S. Goolaup, N. Singh, C. Spezzani, M. Sacchi, J. Phys. D 41 (2008) 134014.

[18] P. Vavassori, F. Spizzo, E. Angeli, D. Bisero, F. Ronconi, J. Magn. Magn. Mater. 262 (2003) 120.

[19] V.G. Harris, K.D. Aylesworth, B.N. Das, W.T. Elam, N.C. Koon, Phys. Rev. Lett. 69 (1992) 1939.

[20] F. Hellman, E.M. Gyorgy, Phys. Rev. Lett. 68 (1992) 1391.

[21] R.E. Camley, R.L. Stamps, J. Phys. Condens. Matter 5 (1993) 3727.

[22] Y. Mimura, N. Imamura, T. Kobayashi, A. Okada, Y. Kushiro, J. Appl. Phys. 49 (1978) 1208.

[23] J.L. Weston, A. Butera, T. Lograsso, M. Shamsuzzoha, I. Zana, G. Zangari, J. Barnard, IEEE Trans. Magn. 38 (2002) 2832. 